\title{
Temperature field at the twin-roll casting of aluminium alloys: computational model and measurements
}

\author{
H. Gjerkeš, S. Hartman, R. Vertnik \& B. Šarler \\ Laboratory for Multiphase Processes, University of Nova Gorica, \\ Slovenia
}

\begin{abstract}
Industrial twin-roll casting of aluminium alloys requires optimisation of connections between process parameters and product properties. To a large extent, the cast strip properties depend on the spatial and temporal characteristics of heat transfer from the cast strip, which needs estimation of the influence of process parameters on the temperature field and temperature gradients in a strip for various alloys, which are cast on an industrial machine. For this purpose, the recently developed meshless Local Radial Basis Function Collocation Method (LRBFCM) was used in the simulation of heat transfer from the strip. The solution of the nonlinear convection-diffusion equation is based on a mixture continuum formulation of the energy transport in solid-liquid phase change systems. Simulation results were compared with experimental values, which were obtained during the industrial production process. They were measured in situ by the specially designed apparatus with fast response thermocouples, which can measure the time-dependent temperature of the cast strip in several locations on the moving strip surface simultaneously. The time-dependent measurement was coupled with a digital camera recording to gather the spatial domain measurement of temperature. The differences between the numerical simulation and experimental results were smaller than $6 \mathrm{~K}$. An explanation for the discrepancy is given.
\end{abstract}

Keywords: twin-roll casting, aluminium alloys, measurements, surface temperature, meshless method, radial basis functions. 


\section{Introduction}

The twin-roll continuous casting is a promising commercial material processing technique, however it is extremely difficult to control [1]. Process modelling [2] has been introduced in order to improve quality, reduce production cost, and improve safety. Like most commercial material processes, the twin roll casting involves many interacting phenomena of great complexity, which makes it difficult to include them all in the model. The principal aim of the presented model is to predict the product properties as a function of the process parameters.

A broad class of meshfree methods in development today are based on Radial Basis Functions (RBFs). The RBF collocation method or Kansa method is the simplest of them. This paper describes the numerical solution of the convectivediffusive solidification problem in twin-roll casting process by the recently developed meshless local radial basis function collocation approach [3-5]. The method is structured on multiquadrics radial basis functions. Instead of global, the collocation is made locally over a set of overlapping domains of influence and the time-stepping is performed in an explicit way. Only small systems of linear equations with the dimension of the number of nodes included in the domain of influence have to be solved for each node. The computational effort grows roughly linearly with the number of the nodes. The developed approach overcomes the principal large-scale problem bottleneck of the original Kansa method.

Simulation results were compared with the measured temperatures on the cast strip surface after exiting the rolls. Presented experimental technique enables temperature measurements during the industrial continuous twin-roll casting without disturbing the production process. The technique is based on fast response thermocouples, mounted on the cast strip using the specially designed apparatus. The time-dependent temperature measurement on the moving cast strip was coupled with digital camera recording to obtain the measurement of surface temperature over a substantial part of the strip exiting the cooling rolls. Influence of the experimental set-up on the measurement results is considered. Based on experimental results, the improvements of the numerical model boundary conditions are proposed.

\section{Model}

The twin-roll casting process, fig. 1, consists of two oppositely rotating internally water cooled rolls which are independently driven. The molten metal is fed between the rolls through a pouring nozzle. The strip thickness is defined with the separation distance between rolls, which is typical few millimetres at strip width of a meter or more.

The heat transfer in twin-roll casting can be reasonably represented in the framework of the mixture continuum formulation which assumes local thermodynamic equilibrium between the phases. This formulation can in solidification context involve quite complicated constitutive relations. Presented model focuses on a convective-diffusive heat transport as a first step. 


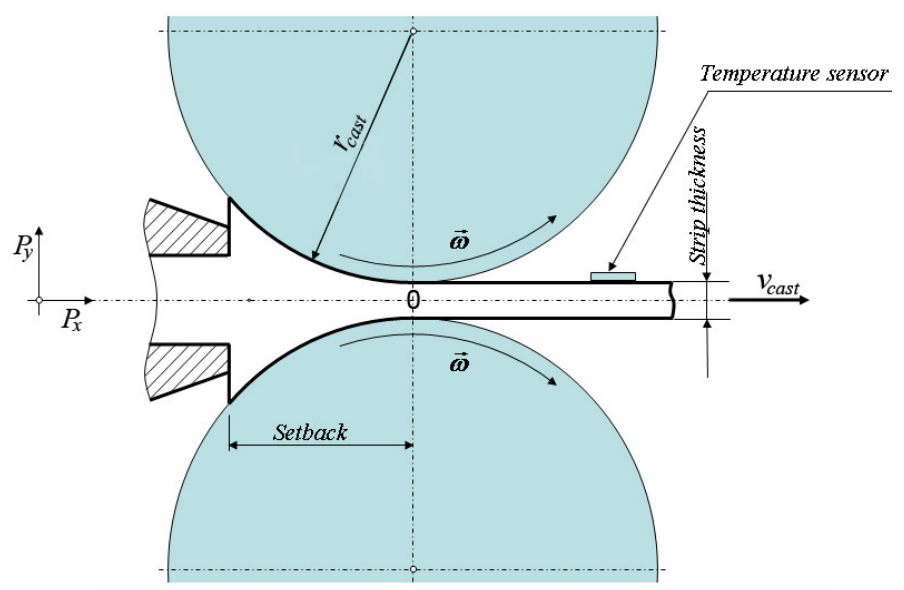

Figure 1: Twin-roll casting process.

\subsection{Governing equations}

Consider a connected fixed domain $\Omega$ with boundary $\Gamma$ occupied by a phase change material described with the temperature dependent density $\rho_{\wp}$ of the phase $\wp$, temperature dependent specific heat at constant pressure $c_{p}$, effective thermal conductivity $k$, and the specific latent heat of the solid-liquid phase change $h_{m}$. The mixture continuum formulation of the enthalpy conservation for the assumed system is

$$
\frac{\partial}{\partial t}(\rho h)+\nabla \cdot(\rho \vec{v} h)=\nabla \cdot(k \nabla T)+\nabla \cdot\left(\rho \vec{v} h-f_{S}^{V} \rho_{S} \vec{v}_{S} h_{S}-f_{L}^{V} \rho_{L} \vec{v}_{L} h_{L}\right)
$$

with subscripts $S$ and $L$ denoting the solid and the liquid phase, respectively. The mixture density is defined as $\rho=f_{S}^{V} \rho_{S}+f_{L}^{V} \rho_{L}$, the mixture velocity is defined as $\rho \vec{v}=f_{S}^{V} \rho_{S} \vec{v}_{S}+f_{L}^{V} \rho_{L} \vec{v}_{L}$, and the mixture enthalpy is defined as $h=f_{S}^{V} h_{S}+f_{L}^{V} h_{L}$. The constitutive mixture temperature - mixture enthalpy relationships are

$$
h_{S}=\int_{T_{r e f}}^{T} c_{S} d T, h_{L}=h_{S}(T)+\int_{T_{S}}^{T}\left(c_{L}-c_{S}\right) d T+h_{m}
$$

with $T_{r e f}$ and $T_{S}$ standing for the reference temperature and solidus temperature, respectively. Thermal conductivity and specific heat of the phases can arbitrarily depend on temperature. The liquid volume fraction $f_{L}^{V}$ is assumed to vary from 0 to 1 between solidus $T_{S}$ and liquidus temperature $T_{L}$. We seek for mixture temperature at time $t_{0}+\Delta t$ by assuming known temperature and velocity fields at time $t_{0}$, and boundary conditions. 


\subsection{Solution procedure}

The solution of the problem is demonstrated on the general transport equation defined on a fixed domain $\Omega$ with boundary $\Gamma$, standing for a reasonably broad spectra of mass, energy, momentum and species transfer problems (and includes also eqn. (1) as a special case):

$$
\frac{\partial}{\partial t}[\rho \mathrm{C}(\Phi)]+\nabla \cdot[\rho \vec{v} \mathrm{C}(\Phi)]=-\nabla \cdot(-\mathbf{D} \nabla \Phi)+S
$$

with $\rho, \Phi, t, \vec{v}, \mathbf{D}$, and $S$ standing for density, transport variable, time, velocity, diffusion matrix and source, respectively. Scalar function $\mathrm{C}$ stands for possible more involved constitutive relations between conserved and diffused quantities. The solution of the governing equation for the transport variable at the final time $t_{0}+\Delta t$ is sought, where $t_{0}$ represents the initial time and $\Delta t$ the positive time increment. The solution is constructed by the initial and boundary conditions that follow. The initial value of the transport variable $\Phi(\vec{p}, t)$ at point with position vector $\vec{p}$ and time $t_{0}$ is defined through the known function $\Phi_{0}$ :

$$
\Phi(\vec{p}, t)=\Phi_{0}(\vec{p}) ; p \in \Omega+\Gamma .
$$

The boundary $\Gamma$ is divided into not necessarily connected parts $\Gamma=\Gamma_{D} \cup \Gamma_{N} \cup \Gamma_{R}$ with Dirichlet, Neumann and Robin type boundary conditions, respectively. These boundary conditions are at the boundary point $\vec{p}$ with normal $\vec{n}_{\Gamma}$ and time $t_{0}<t \leq t_{0}+\Delta t$ defined through known functions $\Phi_{\Gamma}^{D}$, $\Phi_{\Gamma}^{R}, \Phi_{\Gamma r e f}^{R}:$

$$
\Phi=\Phi_{\Gamma}^{D} ; \vec{p} \in \Gamma_{D}, \frac{\partial}{\partial n_{\Gamma}} \Phi=\Phi_{\Gamma}^{N} ; p \in \Gamma_{N}, \frac{\partial}{\partial n_{\Gamma}} \Phi=\Phi_{\Gamma}^{R}\left(\Phi-\Phi_{\Gamma r e f}^{R}\right) ; p \in \Gamma_{R} .
$$

The involved parameters of the governing equation and boundary conditions are assumed to depend on the transport variable, space and time. The solution procedure is in this paper based on the combined explicit-implicit scheme. The discretisation in time can be written as

$$
\frac{\partial}{\partial t}(\rho \mathrm{C}(\Phi)) \approx \frac{\rho \mathrm{C}-\rho_{0} \mathrm{C}_{0}}{\Delta t} \approx \frac{\bar{\rho} \overline{\mathrm{C}}+\bar{\rho} \frac{d \bar{C}}{d \Phi}(\Phi-\bar{\Phi})-\rho_{0} \mathrm{C}_{0}}{\Delta t}
$$

by using the two-level time discretisation and Taylor expansion of the function $\mathrm{C}(\Phi)$. The known quantities are denoted with overbar. The source term can be expanded as

$$
S(\Phi) \approx \bar{S}+\frac{d \bar{S}}{d \Phi}(\Phi-\bar{\Phi})
$$

The unknown $\Phi$ can be calculated from the equation 


$$
\Phi=\frac{\frac{\rho_{0}}{\Delta t} \mathrm{C}_{0}-\frac{\bar{\rho}}{\Delta t} \overline{\mathbf{C}}+\frac{\bar{\rho}}{\Delta t} \frac{d \bar{C}}{d \Phi} \bar{\Phi}+\nabla \cdot\left(\mathbf{D}_{0} \nabla \Phi_{0}\right)-\nabla \cdot\left(\rho_{0} \vec{v}_{0} \mathrm{C}_{0}\right)+\bar{S}-\frac{d \bar{S}}{d \Phi} \bar{\Phi}}{\frac{\bar{\rho}}{\Delta t} \frac{d \bar{C}}{d \Phi}-\frac{d \bar{S}}{d \Phi}}
$$

The value of the transport variable $\Phi_{n}$ is solved in as set of nodes $\vec{p}_{n} ; n=1,2, \ldots, N$ of which $N_{\Omega}$ belong to the domain and $N_{\Gamma}$ to the boundary. The iterations over one timestep are completed when the equation (12) is satisfied, and the steady-state is achieved when the eqn. (13) is achieved

$$
\max \left|\Phi_{n}-\bar{\Phi}_{n}\right| \leq \Phi_{i t r}, \max \left|\Phi_{n}-\Phi_{0}\right| \leq \Phi_{s t e} .
$$

The value of the unknown derivatives of the variable $\Phi_{n}$ in point $\vec{p}_{n}$ is approximated by the collocation method which uses the values of $\Phi_{i}$ at $I$ points $\vec{p}_{i} ; i=1,2, \ldots, I$, situated in the vicinity of and including $\vec{p}_{n}$. One can write the following approximation of the function and its first and second order partial derivatives

$$
\begin{gathered}
\Phi(\vec{p}) \approx \sum_{k=1}^{K} \alpha_{k} \psi_{k}\left(\vec{p}-\vec{p}_{n}\right), \frac{\partial}{\partial p_{\varsigma}} \Phi(\vec{p}) \approx \sum_{k=1}^{K}{ }_{n} \alpha_{k} \frac{\partial}{\partial p_{\varsigma}} \psi_{k}\left(\vec{p}-\vec{p}_{n}\right) \\
\frac{\partial^{2}}{\partial p_{\varsigma \xi}} \Phi(\vec{p}) \approx \sum_{k=1}^{K} \alpha_{k} \frac{\partial^{2}}{\partial p_{\varsigma \xi}} \psi_{k}\left(\vec{p}-\vec{p}_{n}\right) ; \quad \varsigma, \xi=x, y .
\end{gathered}
$$

The scaled multiquadrics are used for representation functions

$$
\psi_{k}\left(\vec{p}-\vec{p}_{n}\right)=\left[\left(\vec{p}-\vec{p}_{n}\right)^{2}-c^{2} r_{0}^{2}\right]^{1 / 2}
$$

where $r_{0}$ represents the maximum distance between points in a subdomain. Other details of the solution (particularly implementation of boundary conditions) are elaborated in [3-5].

\subsection{Model results}

The aluminium alloy composition and the nominal process parameters, used as simulation input parameters, are given in tables 1 and 2, respectively.

Table 1: Aluminium alloy composition.

\begin{tabular}{|l|l|l|l|l|l|l|l|l|l|}
\hline El. & $\mathrm{Fe}$ & $\mathrm{Si}$ & $\mathrm{Mn}$ & $\mathrm{Mg}$ & $\mathrm{Cu}$ & $\mathrm{Zn}$ & $\mathrm{Ti}$ & $\mathrm{Cr}$ & $\mathrm{B}$ \\
\hline $\mathrm{Wt}[\%]$ & 0.85 & 0.6 & 0.022 & 0.05 & 0.05 & 0.05 & 0.0375 & 0.002 & 0.01 \\
\hline
\end{tabular}

Table 2: $\quad$ Process and input parameters.

\begin{tabular}{|l|l|}
\hline Casting Speed $[\mathrm{m} / \mathrm{min}]$ & 1.22 \\
\hline Strip thickness $[\mathrm{mm}]$ & 6.00 \\
\hline Roll temperature $\left[{ }^{\circ} \mathrm{C}\right]$ & 35.1 \\
\hline Setback $[\mathrm{mm}]$ & 69.22 \\
\hline Heat transfer coefficient strip-cooling rolls $\left[\mathrm{W} /\left(\mathrm{m}^{2} \mathrm{~K}\right)\right]$ & 3600 \\
\hline Convection heat transfer coefficient strip-air $\left[\mathrm{W} /\left(\mathrm{m}^{2} \mathrm{~K}\right)\right]$ & 10.7 \\
\hline
\end{tabular}




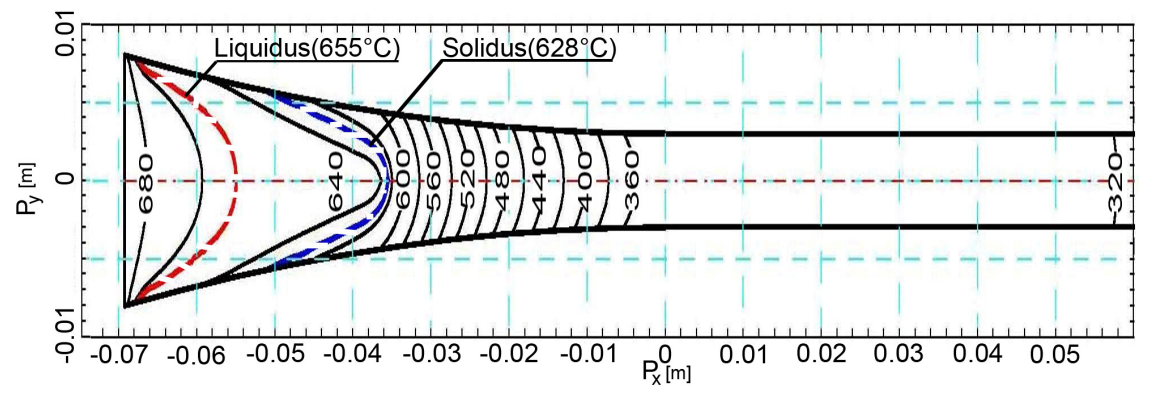

Figure 2: Predicted temperature field in cast strip cross-section at nominal process parameters.

Fig. 2 shows prediction of the temperature field in cast aluminium alloy strip cross-section at the process parameters, which were used at the time of the measurements, presented below. More simulation results for temperature field in the cast strip as a function of various process parameters: casting speed, strip thickness, roll temperature, setback, etc., can be found in [3-5].

\section{Experimental set up}

A new measurement technique was developed for surface temperature measurements of twin-roll cast aluminium alloys. The fast response Omega CO1 Type $\mathrm{K}$ thermocouples with butt welded junction of thickness $0.013 \mathrm{~mm}$ were used. The thermocouple is embedded between two thin, glass reinforced high temperature polymer laminates for support and electrical insulation. The laminated sensor has dimensions $19 \times 9.4 \times 0.13 \mathrm{~mm}$. Declared response time is between 10 and $20 \mathrm{~ms}$, when grounded to surface. The sensor was installed on a small, $12 \times 8 \times 5 \mathrm{~mm}$ graphite block with a $2 \mathrm{~mm}$ thick layer of temperature resistant silicone putty. The latter was used to reduce the influence of the transient heat conduction in the thermocouple support on the dynamic temperature measurements, i.e. to improve the response of the sensor. Thermocouples were fastened via graphite blocks to a specially constructed holder. Gravity of the holder assured good contact between thermocouples and measured surface, whereby it could be placed down and removed from the strip surface without disturbing or influencing the production process. The detail of the thermocouple fixation and the set up, placed on the cast strip during the measurement are shown in fig. 3 .

Measurement procedure started with placing down the holder with thermocouples on the upper cast aluminium alloy strip surface as near as possible to the cast rolls, typically $100 \mathrm{~mm}$ from the rolls' outlet (point 0 in fig. 1). Thermocouples travelled together with cast strip without relative moving. The temperatures were sampled using the high-speed data acquisition system Agilent 34970A with multiplexer module 34902A. Simultaneously, the position of the thermocouples was recorded with a digital camera, whereby clocks in the camera, the data acquisition system and the process control system were synchronised. 


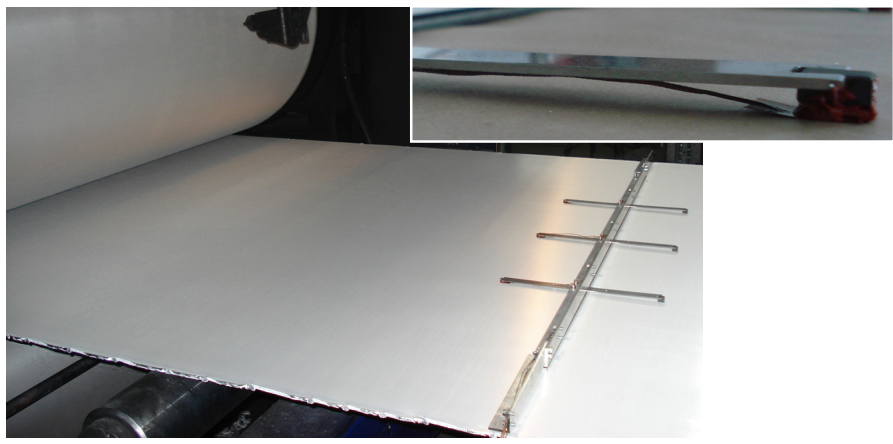

Figure 3: The set up during measurement (left bottom) and thermocouple installation detail (right top).

Information from digital camera recording enabled conversion of the timedependent into spatial-dependent measurement of the strip surface temperature.

The specially designed apparatus gives possibility to measure strip surface temperature during the industrial continuous twin-roll casting of aluminium alloys, where no influences of the measurement procedure on the production process are allowed. On the other hand, the thermocouple installation on the graphite block deteriorates the sensor dynamic response because of the additional thermal inertia, which increases the delay time of the sensor. The influence of the thermocouple installation support was considered with the heat flow balance of the sensor:

$$
\left(T_{A}-T_{m}\right) \alpha_{A m} A=(\rho c V)_{m} \frac{d T_{m}}{d t}+Q_{1}
$$

where $T_{A}$ is the surface temperature of the aluminium strip, $T_{m}$ is the measured temperature, $\alpha_{A m}$ is the coefficient describing the heat transfer between the aluminium surface and sensor, $A$ is the sensor contact area, $(\rho c V)_{m}$ is the thermal capacitance of the sensor, and $Q_{1}$ is heat flow from the sensor to the silicone layer and graphite installation support. Defining the thermocouple time constant as $t_{m}=\frac{(\rho c V)_{m}}{\alpha_{A m} A}$, eqn. (18) can be rearranged to express surface temperature measurement error:

$$
\left(T_{A}-T_{m}\right)=t_{m} \frac{d T_{m}}{d t}+\frac{q_{1}}{\alpha_{A m}} .
$$

Time derivatives of the measured temperature were calculated with the first order central difference scheme. The thermocouple time constant $t_{m}$ was determined in separated test runs to be $3 \mathrm{~s}$ and should be the same for the all measurement runs, as well as the fitting coefficient $\alpha_{A m}$.

The heat flux from measurement sensor to the silicone layer $q_{1}$ was calculated using the equations describing the response of a semi-infinite solid to a step input of heat flux $q_{l}$ at the surface of the silicone layer [6]. Subtracting the 
temperature variation at the surface between the sensor and silicone layer from temperature variation at silicone layer depth $l_{S l}$ with temperature $T_{G}$ gives the expression for variation of $q_{1}$ :

$$
q_{1}=\frac{\left(T_{m}-T_{G}\right) k_{S i}}{l_{S i}\left[\sqrt{\frac{\tau}{\pi}}\left(1-e^{-\frac{1}{\tau}}\right)+\operatorname{erfc}\left(\frac{1}{\sqrt{\tau}}\right)\right]}
$$

where $T_{G}$ is measured temperature at the back of the graphite support block with the same kind of the thermocouple as $T_{m}, \tau=\frac{4 \kappa_{S l} t}{l_{S l}^{2}}$ is the dimensionless time for the silicone layer of thickness $l_{S l}$ and of thermal diffusivity $\kappa_{S l}=\frac{k_{S l}}{\rho_{S l} c_{S l}} \cdot \rho_{S l}, c_{S l}$, and $V_{S l}$ are density, specific heat and volume of the silicone layer, respectively.

\subsection{Experimental results}

The difference between the actual and measured temperature of the cast aluminium strip surface, $T_{A}-T_{m}$, is calculated with eqn. (19) for four measurement runs at the same process parameters and shown in fig. 4 in dependence of the sensor installation support starting temperature (runs $\# 30$ and $\# 32$ : $50^{\circ} \mathrm{C}$; runs $\# 31$ and \#33: $210^{\circ} \mathrm{C}$ ). At the beginning, the differences are the largest, from 10 to $22 \mathrm{~K}$, and are falling to $2.5 \mathrm{~K}$ in all runs at the end of the measurement, which was limited to about $1.9 \mathrm{~m}$ or $90 \mathrm{~s}$. In fig. 5 , the measured, $T_{m}$, and the actual, $T_{A}$, time dependent strip surface temperatures are shown for two runs. It is evident, that $T_{A}$, calculated with eqn. (19), is independent of $T_{m}$ starting temperature.

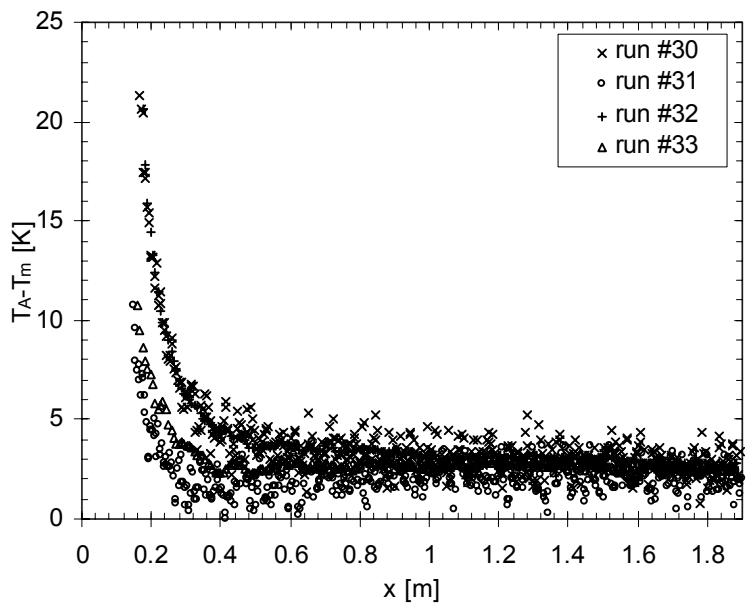

Figure 4: Difference between measured and actual strip surface temperature, spatial coordinate. 


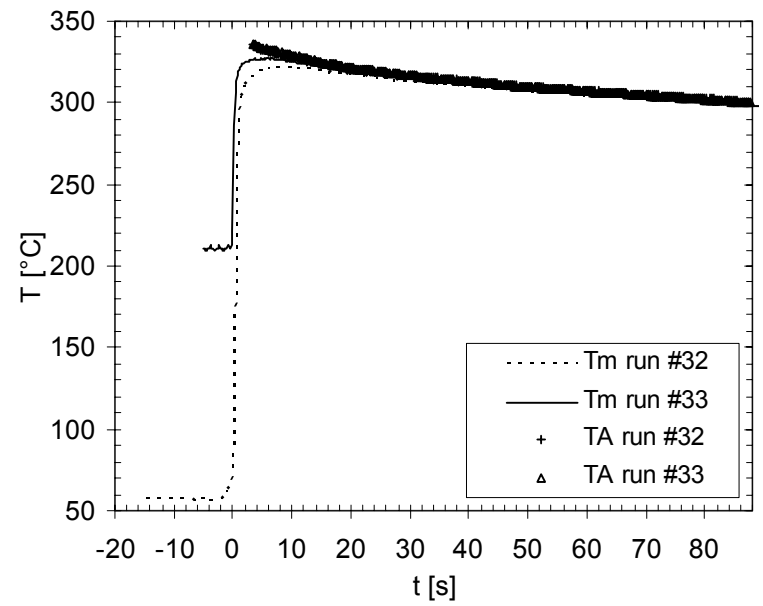

Figure 5: $\quad$ Measured and actual strip surface temperature, time coordinate.

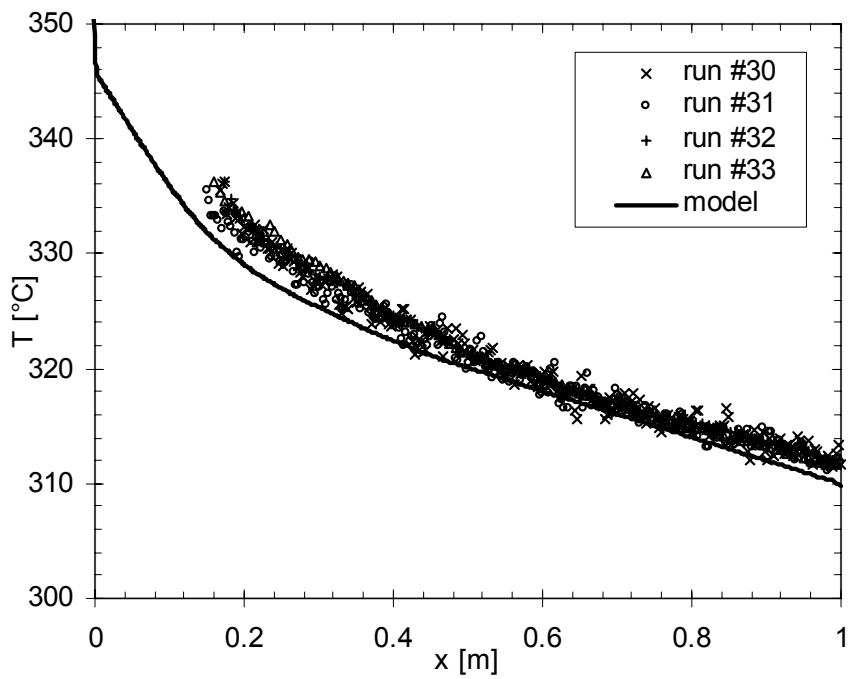

Figure 6: Comparison of the simulated and measured strip surface temperature in four runs.

\section{Experimental validation of the model}

Results of the predicted temperature field of the cast aluminium strip crosssection were compared with the measured temperature on the upper side in the middle of the cast strip surface. Standard deviation of $T_{A}$ for all runs is $1.3 \mathrm{~K}$.

Fig. 6 shows good agreement between simulation and measurements with maximum discrepancy of $6 \mathrm{~K}$ near the cooling rolls. Comparison indicates overestimation of the convection heat transfer between strip and surrounding air, 
and need for the radiation heat transfer consideration between cold rolls and strip surface, which has large influence on strip cooling in the vicinity of the rolls.

\section{Conclusion}

The meshless Local Radial Basis Function Collocation Method (LRBFCM) was used for estimation of the process parameters influence on the temperature field in the industrial twin-roll continuous casting aluminium alloy strip in the simulation of heat transfer from the strip. The model can consider influence of various process parameters on the temperature of the cast strip, which determines its properties. Simulation results were in good agreement with in situ measurements during the production process with the developed measurement technique. In addition, the experimental results gave important information for the model improvement, especially at the definition of the boundary conditions.

Mounting three or five temperature sensors in a row and positioning the thermocouples on the same spots on the upper and the lower cast strip surface will represent next steps in development of the presented measurement technique, which will enable measurement of the possible temperature gradient in the width and the transverse sections of the cast strip, which could be responsible for inhomogeneous material structure and consecutive, difficulties at further strip processing.

\section{References}

[1] D. G. Altenpohl, Aluminum: Technology, Applications, and Environment: a Profile of a Modern Metal, Aluminium Association \& TMS, 1998.

[2] T. Saitoh, H. Hojo, H. Yaguchi and C.K. Kang, Two dimensional model for twin roll continuous casting”, Metallurgical Transactions, 20B, pp.381-390 1989.

[3] B. Šarler, R. Vertnik \& S. Šaletić, Solution of the thermal model of the twin-roll casting process by the meshless local radial basis function collocation technique. Computational methods for coupled problems in science and engineering, eds. M. Papadrakakis, E. Onate \& B. Schrefler, Barcelona: International Center for Numerical Methods in Engineering (CIMNE), 2005.

[4] B. Šarler \& R. Vertnik, Meshfree explicit radial basis function collocation method for diffusion problems, Computers and Mathematics with Applications, 51, pp. 1269-1282, 2006.

[5] R. Vertnik \& B. Sarler, Meshless local radial basis function collocation method for convective-diffusive solid-liquid phase change problems, International Journal of Numerical Methods for Heat and Fluid Flow, 16, pp. 617-640, 2006.

[6] B. Lawton \& G. Klingerberg, Transient Temperature in Engineering and Science, Oxford University Press, 1996. 